\title{
Simulation, Measurement and Modeling of Orthogonal On-Chip Interconnects
}

\author{
Yves Quéré, Thierry Le Gouguec, Pierre-Marie Martin, Denis Le Berre, and Fabrice Huret \\ LEST-UMR CNRS n ${ }^{\circ} 6165$-CS 93837-29238 Brest Cedex 3-France \\ Phone: +33 (0)2-98-01-82-73; Fax: +33(0)2-98-01-63-95; Email: yves.quere@ univ-brest.fr
}

\begin{abstract}
This paper explains the appearance of resonant frequency due to underlayer orthogonal metal grid in microstrip line structures. In the context of global interconnects, this resonant frequency may lead to noise and has to be estimate. A model, which allows one to approximate this resonant frequency, is validated by measurement.
\end{abstract}

\section{INTRODUCTION}

A feature of evolution in Microelectronics is the marked enhancement of integration- and circuits-running speeds. A trend in modern high speed CMOS VLSI circuits is the reduction of features sizes together with the increase of chip dimensions. Today, the race towards integration is slowed down by the problem of global interconnects, which constitutes one of the blocking points in the improvement of circuits performances. Further to the elevation of operating frequencies in high-speed digital circuits, propagation delays are affected by coupling noise [1]-[2].

In CMOS technology circuits, the transistors are connected by copper lines at various metal levels. Generally, all the copper conductors placed on a same level are parallel, whereas the lines are orthogonally built on the closer level. On-chip high-speed interconnects with underlayer orthogonal metal grids, including grid-backed lines (GBLs) and grid-backed coplanar waveguide (GBCPWs), have been studied in recent papers [3]-[8]. A significant enhancement of transmission characteristics due to the presence of underlayer orthogonal metal grid have been demonstrated in the case of GBLs [7]-[8] and a weaker effect has been observed for coplanar interconnects [7]. Moreover, a resonant frequency has been observed in the case of coplanar and microstrip lines [7].

In this paper, we explain the appearance of resonant frequency due to orthogonal metal grids in coplanar and microstrip line structures. Simulations and measurements, which illustrate these frequencies, are presented in section II. A model which allows one to approximate the resonant frequency is proposed and validated in section III.

\section{SIMULATION AND EXPERIMENTAL VALIDATION}

In this part, microstrip and coplanar structure with underlayer metal grid are presented. The simulation of these structures has been made with the electromagnetic solver Momentum (Agilent) in the 1-50 GHz frequency range. TRL calibration has been used for measurements. Structures with various grid densities have been realized. We study two different cases of grid, floating or connected to the ground. In this paper, we only present the study results of microstrip line structures. A measurement result of coplanar lines will be illustrated in the conclusion.
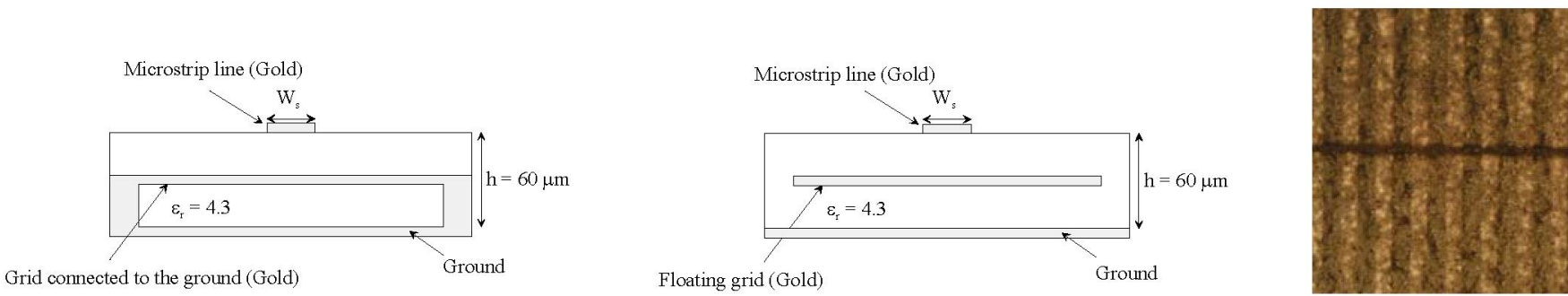

Fig. 1. Cross-section of microstrip line structure with connected grid (on the left) and floating grid (on the middle); top view picture of the realized microstrip structure (on the Right). ( $\left.W_{s}=30 \mu \mathrm{m}\right)$ 

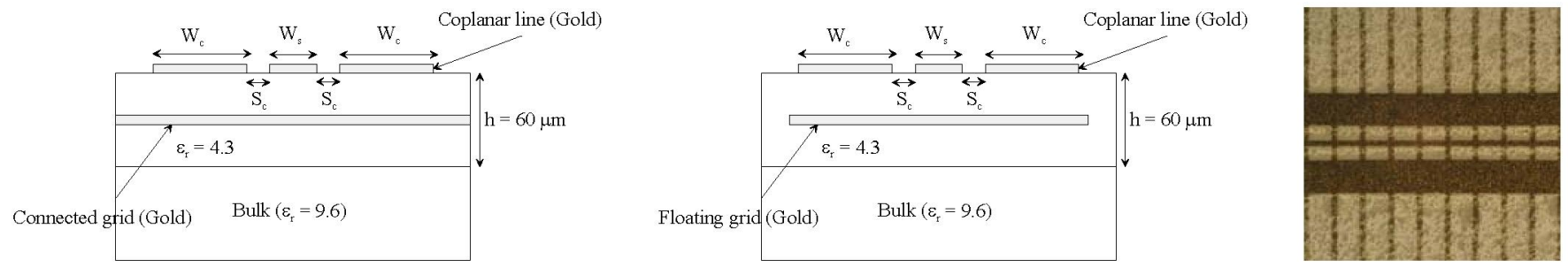

Fig. 2. Cross-section of coplanar line structure with connected grid (on the left) and floating grid (on the middle); top view picture of the realized coplanar structure (on the Right). ( $W_{s}=30 \mu \mathrm{m}, W_{c}=170 \mu \mathrm{m}, S_{c}=70 \mu \mathrm{m}$ )

The line length is equal to $1.5 \mathrm{~mm}$, the thickness of metallization is equal to $4 \mu \mathrm{m}$ and the grid is placed on the middle of the substrate. The grid line length is equal to $3.285 \mathrm{~mm}$ when the spacing and width vary in the $50-100 \mu \mathrm{m}$ range. In the presented results, grid line width and space are equal to $50 \mu \mathrm{m}$. The simulated and measured $S$ parameters, in the case of microstrip lines with connected and floating underlayer metal grid, are presented in figure 3 . We note a good agreement between simulations and measures and the presence, in each cases of a resonant frequency. In the case of connected grid, the resonant frequency is equal to $20 \mathrm{GHz}$, and equal to $45 \mathrm{GHz}$ in the case of floating grid. The set of measures and simulations shows that the resonant frequency value is weakly dependent on grid density, number of grid line and space between grid and microstrip line. The resonant frequency especially depends on grid lines length, substrate relative permittivity and grid lines termination.
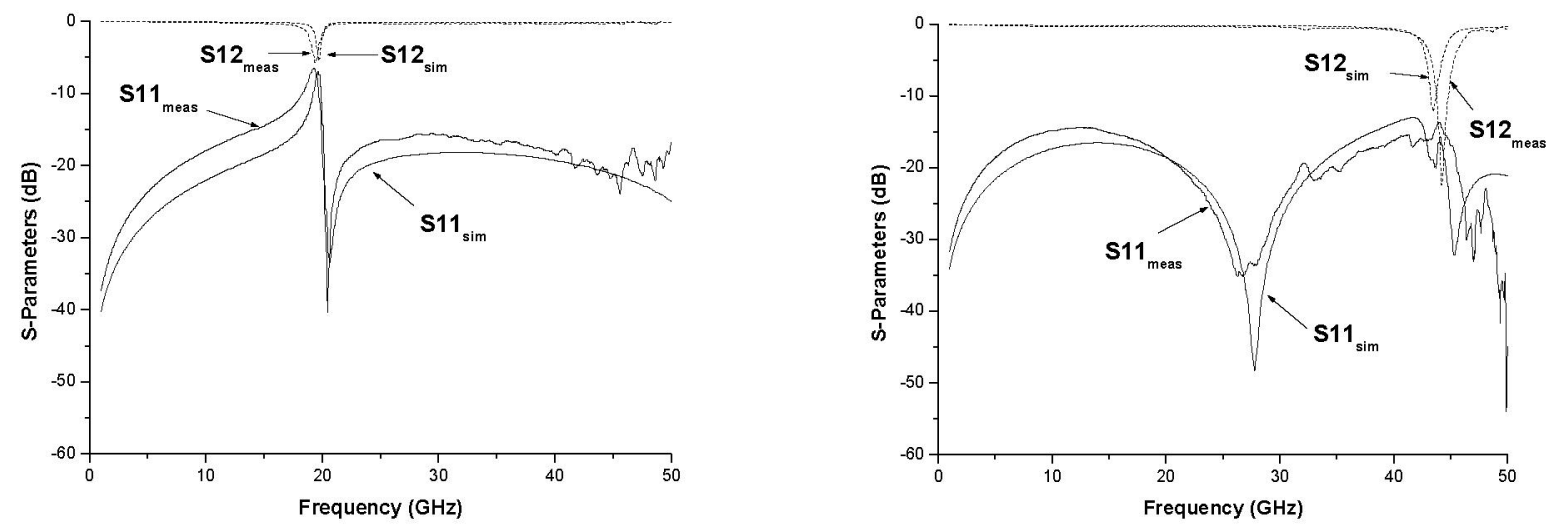

Fig. 3. Simulated and measured $S$-parameters for microstrip line structure with connected grid (on the left) and floating grid (on the right).

The $S$-parameters evolution and the two different values of the resonant frequency (in the case of connected or floating grid) are indications that the structure could be compared to a stub filter used in microwave domain, naturally connected by a capacitance. In the case of stub filters, the resonant frequency of short-circuit stub is twice larger than an open-circuit one. The underlayer metal grid behavior is then opposite to the stub one. In the next section, we will model the metal grid by comparison with the stub in order to find the resonant frequency value.

\section{MODELING AND THEORETICAL. VALIDATION}

Figure 4 presents the microstrip structure model with a connected stub and with an underlayer metal line. $Z_{R}$ is the stub or grid line input impedance, $Z_{L}$ the termination impedance and $C$ the capacitance between the grid and the microstrip line. Circuit simulations, using geometric values of the structure, show (Fig. 5) that considering only one grid line permits to find the measured resonant frequency in the both cases, connected and floating grid line. Moreover, a comparison between the simulated $S$-parameters of the model with stub and the grid line one show an opposite behavior. In deed, the resonant frequency of a parallel short-circuit stub (Fig. 4) is closed to the floating grid one. Reciprocally, the resonant frequency of a parallel open-circuit stub is closed to a connected grid line one. 

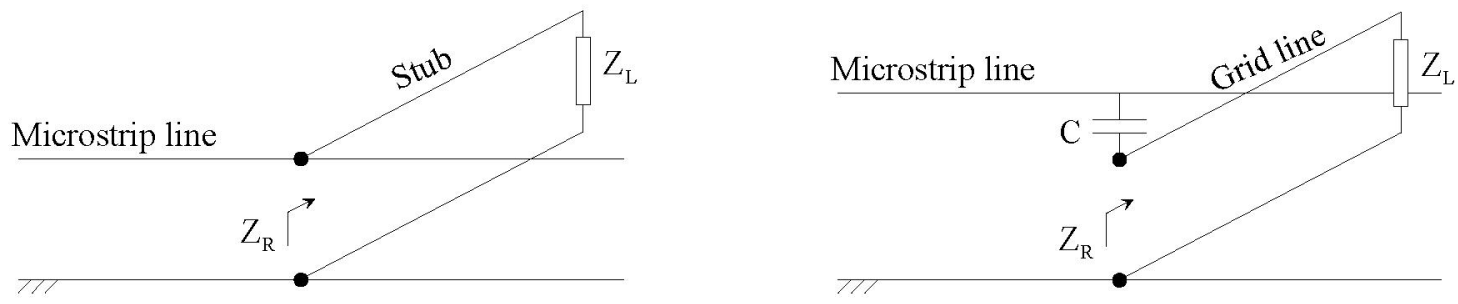

Fig. 4. Stub (on the left) and grid line (on the right) models
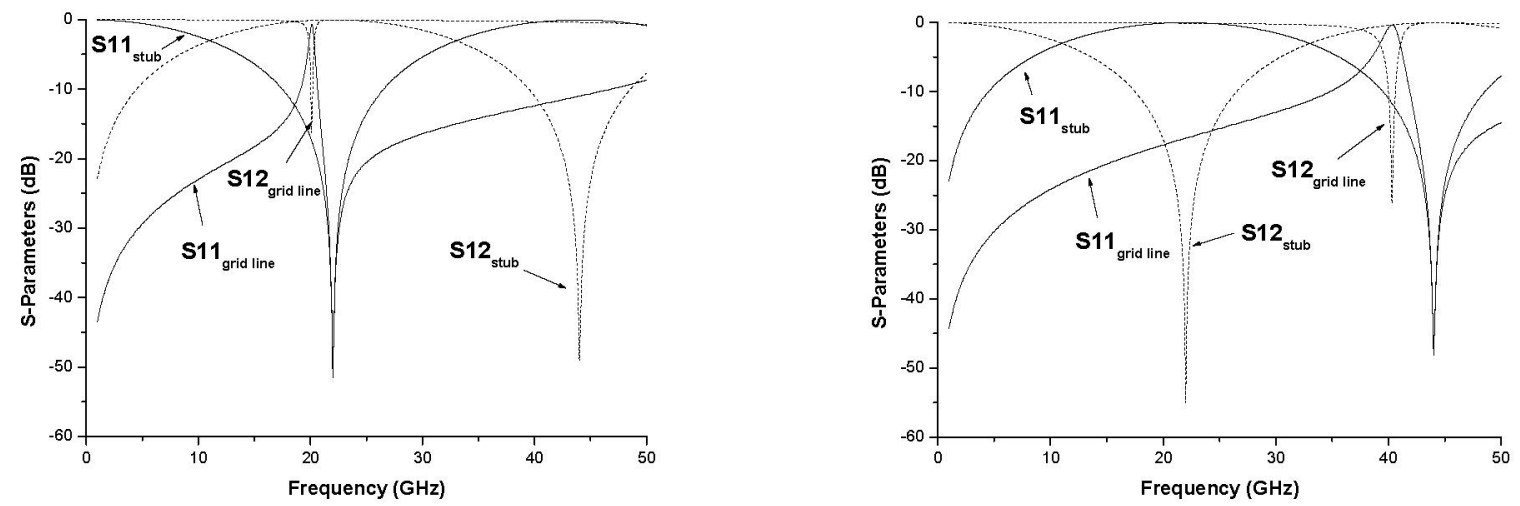

Fig. 5. Simulated $S$-parameters with stub or underlayer grid line terminated by short-circuit (on the left) and open-circuit (on the right).

Input stub impedance calculation permits to obtain the resonant frequency $f_{R}$ of a stub. Input stub impedances and associated resonant frequencies are given in equations (1)-(2) and (3)-(4) for short- and open-circuit termination respectively. The input stub impedance is called $Z_{R}, Z_{c}$ is the stub characteristic impedance, $\beta$ the phase factor, $l$ the stub length, $\varepsilon_{\text {reff }}$ the effective relative permittivity of the substrate and $c$ the light velocity.
(1) $Z_{R}=\frac{j Z_{c} \operatorname{tg}(\beta l)}{2}$
(2) $f_{R}=\frac{c}{2 l \sqrt{\varepsilon_{\text {reff }}}}$
(3) $Z_{R}=\frac{Z_{c}}{2 j \operatorname{tg}(\beta l)}$
(4) $f_{R}=\frac{c}{4 l \sqrt{\varepsilon_{r e f f}}}$

In the case of perpendicular grid line, the input impedance $Z_{R}$ has to be added to the capacitance $C$. This new impedance is called $Z_{p}$ in equations (5) and (6) where equation (5) corresponds to a connected grid structure and equation (6) a floating one.
(5) $Z_{p}=\frac{1}{j C \omega}+\frac{Z_{c}}{2} \frac{Z_{R}+j Z_{c} \operatorname{tg}(\beta l)}{Z_{c}+Z_{R} \operatorname{tg}(\beta l)}$
(6) $Z_{p}=j\left(-\frac{1}{C \omega}+\frac{Z_{c} \operatorname{tg}(\beta l)}{2}\right)$

The resonant frequency appears when the impedance $Z_{p}$ is equal to zero, ie when relations (7) and (8) are respected.
(7) $\beta l=\operatorname{arctg}\left(\frac{2}{Z_{c} C \omega}\right)$
(8) $\beta l=\operatorname{arctg}\left(-\frac{Z_{c} C \omega}{2}\right)$

The capacitance $C$ is, generally, very weak (near to $20 \mathrm{fF}$ in our case of concern). Then, it is possible to approximate in a first order the resonant frequency $f_{R}$ by equation (9) for a connected grid and equation (10) for a floating one. 
These resonant frequencies depend on the grid line length and on the effective relative permittivity. In deed, any decrease in effective relative permittivity and grid line length involves an increase in resonant frequency value. Consequently, the noise caused by underlayer orthogonal metal grid is reduced.

$$
\text { (9) } f_{R}=\frac{c}{4 l \sqrt{\varepsilon_{\text {reff }}}}
$$

$$
\text { (10) } f_{R}=\frac{c}{2 l \sqrt{\varepsilon_{\text {reff }}}}
$$

\section{CONCLUSION}

In this paper, we evidenced by simulation and measurement, in the case of microstrip line, a resonant frequency due to orthogonal metal layer. We showed that this resonant frequency can be easily approximate and we demonstrated that connected grid involves in a resonant frequency twice weakly than a floating grid. Moreover, the resonant frequency value especially depends on the length of orthogonal lines and on the relative permittivity of the substrate. Its also depends on the capacity between the grid and the microstrip or coplanar lines, but its effect is weaker due to its low value. We also measured the $S$-parameters of a coplanar structure with an underlayer orthogonal metal grid (Fig. 6). These measures also show the appearance of resonant frequencies due to this orthogonal layer, but which appears at lower frequencies due to a different equivalent model. The later has been also found and permits to approximate the resonant frequency of a coplanar line structure.

A work prospect is the evaluation of the resonant frequency influence on the transmission of high-speed digital signals.

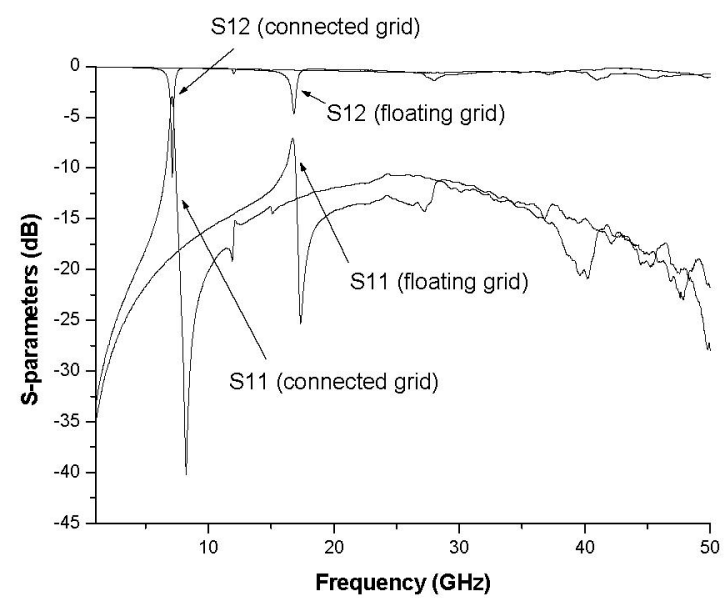

Fig. 6. Measured $S$-parameters with underlayer orthogonal metal grid connected and floating for the coplanar structure.

\section{REFERENCES}

[1] A. Deutsch, P. W. Coteus, and G. V. Kopscay, "On-chip wiring design challenges for gigahertz operation," Proc. IEEE, vol. 89, no. 4, pp. 529-555, Apr. 2001.

[2] S. Morton, "On-chip inductance issues in multiconductor systems," Proc. Design Automation Conference, pp. 921-926, Jun. 1999.

[3] Y. Quéré, T. Le Gouguec, P.M. Martin, and F. Huret, "Interconnect Mode Conversion In High-Speed VLSI Circuits," IEEE International Symposium on Quality Electronic Design (ISQED), San Jose (USA), pp. 265-270, Mar. 2004.

[4] L. David, C. Crégut, F. Huret, Y. Quéré, F. Nyer "Return Path Assumption Validation for Inductance Modeling in Digital Design" IEEE workshop on Signal Propagation on Interconnects, May 2005.

[5] Y. Quéré, T. Le Gouguec, P.M. Martin, D. Le Berre, F. Huret, L. David, and C. Crégut,"3-D Frequency-Dependent RLC Elements Extraction by Full Wave Analysis : Identification of the Return Current Paths in Complex Power Ground Grids of High Speed VLSI Circuits" IEEE workshop on Signal Propagation on Interconnects, May 2005.

[6] Y. Quéré, T. Le Gouguec, N. Tanguy, P.M. Martin, D. Le Berre, F. Huret,"High-Frequency Effects of Orthogonal Interconnect Layers On Inductance in High-Speed VLSI Circuits". IEEE workshop on Signal Propagation on Interconnects, May 2006.

[7] P. Wang, and E. Chich-Chuan Kan, "High-Speed Interconnects With Underlayer Orthogonal Metal Grids," IEEE Trans. on Advanced Packaging, vol. 27, no. 3, pp. 497-507, Aug. 2004.

[8] D. Lutz, V. K. Tripathi, and A. Weisshaar, "Enhanced Transmission Characteristics of On-Chip Interconnects with Othogonal Gridded Shield," IEEE Trans. On Advanced Packaging, vol. 27, no. 3, pp. 497-507, Aug. 2004. 